\title{
The Cartesian diver and the fold catastrophe
}

\author{
J. Güémez ${ }^{\mathrm{a}}$ \\ Departamento de Física Aplicada, Universidad de Cantabria, E-39005 Santander, Spain \\ C. Fiolhais ${ }^{\text {b) }}$ and M. Fiolhais ${ }^{\mathrm{c})}$ \\ Departamento de Física and Centro de Física Computacional, Universidade de Coimbra, \\ P-3004-516 Coimbra, Portugal
}

(Received 10 January 2002; accepted 8 March 2002)

The motion of the Cartesian diver is studied, both theoretically and experimentally, and interpreted as an example of a fold catastrophe, where the control parameter is the external pressure. (C) 2002 American Association of Physics Teachers.

[DOI: $10.1119 / 1.1477433]$

\section{INTRODUCTION}

The Cartesian diver, an hydrostatic apparatus used by Descartes to illustrate Archimedes' principle, has a long history. It was common in old physics cabinets and remains a nice toy ${ }^{1}$ and an interesting demonstration apparatus. ${ }^{2-4}$ A simple do-it-yourself Cartesian diver consists of an eyedropper with air trapped inside its rubber bulb, placed in a large plastic soda bottle containing water. ${ }^{1}$ The diver floats, but it sinks if the closed bottle is gently squeezed. If the pressure is decreased, the process is reversed and the diver rises up. This behavior is intriguing because it does not occur with normal buoys. If we keep the pressure constant, a small perturbation of the floating diver gives rise to damped oscillations. However, a large perturbation may drive the diver to a certain depth, below which it sinks completely. This behavior is an additional surprise of the Cartesian diver. It is not observed in the usual toys because the bottle is not tall enough.

In our theoretical and experimental studies the diver is a test tube, with air trapped inside, that floats or is submersed in a closed vessel containing water. The external pressure may be varied with a syringe and measured with a mercury manometer. ${ }^{5,6}$ The pressure determines the equilibrium position of the test tube. By increasing the external pressure, the floating tube sinks at some point. Such behavior may be interpreted in the context of catastrophe theory, ${ }^{7,8}$ with the external pressure as the control parameter. For a sufficiently low external pressure, the diver potential has a local minimum and a local maximum, with the minimum corresponding to the floating position. Increasing the pressure causes the maximum and the minimum to move, and at a critical pressure they merge (this point is an inflection point). This behavior corresponds to a "fold catastrophe."

The Cartesian diver may also be examined from the dynamical point of view, keeping the pressure constant. When the diver performs small oscillations about its floating position, it is in the attraction basin of the potential minimum. However, a large perturbation may force the diver to cross the local maximum and force it to sink. The maximum of the potential corresponds to a critical depth, that is, the depth below which the sunk diver will not return to the surface.

Although the experiments are relatively easy to perform, well-designed equipment is needed, especially to control the pressure. Additionally, the vessel has to be high enough to allow the diver to sink without returning to the surface. We designed an original apparatus that is adequate to study the quantitative aspects of the Cartesian diver in undergraduate courses.
In Sec. II we describe the static equilibrium of the diver based on Archimedes' principle, Pascal's principle, and Boyle's law, and present some experimental results. In Sec. III we discuss the diver's potential in connection with catastrophe theory. In Sec. IV we describe the diver's dynamics based on Newton's law and present more experimental results. The conclusions appear in Sec. V.

\section{STATIC EQUILIBRIUM}

A tube of length $L$ not completely filled with water [see Fig. 1(a)] is inverted in a large vessel containing the same liquid [Fig. 1(b)]. The original length of the air column inside the tube is $l_{0}$. After the tube has been inverted inside the water, $l<l_{0}$ is the new length of the air bubble. We denote by $x$ the coordinate of the tube's upper part with respect to the liquid level (this level is practically unchanged). If part of the tube is outside the water, as in Fig. 1(b), $x>0$, and the length of the air column below the liquid surface is $\xi=l-x$. If the tube is totally immersed in water, $x<0$ and $\xi=l$. In Sec. IV we study the dynamics of the diver and obtain the dependence of $x$ with the time. When the tube is in static equilibrium, $x=x_{e}$ and $\xi=\xi_{e}$. If the tube is in static equilibrium with $x_{e}=0$, as in Fig. 1(c), $\xi=\xi_{e}^{*}$.

Let us consider the situation shown in Fig. 1(b), with the tube in static equilibrium. If we neglect the mass of the trapped air (because it is much smaller than the mass of the tube) and use Archimedes' principle, we have

$$
m g=V \rho_{\text {glass }} g=A \xi_{e} \rho g+V\left(1-\frac{x_{e}}{L}\right) \rho g,
$$

where $g=9.8 \mathrm{~m} \mathrm{~s}^{-2}, \quad V \approx(\pi / 4) L\left(d_{\text {ext }}^{2}-d_{\text {int }}^{2}\right)$ is the glass volume, ${ }^{9}$ and $A=(\pi / 4) d_{\text {int }}^{2}$ is the internal cross section of the tube ( $d_{\text {ext }}$ and $d_{\text {int }}$ are the external and internal diameters of the test tube, respectively). The glass density is $\rho_{\text {glass }}$ $=m / V$, where $m$ is the mass of the tube, and $\rho=1 \mathrm{~g} \mathrm{~cm}^{-3}$ is the water density.

In our experiments, the initial pressure [see Fig. 1(b)] is always the atmospheric pressure, $P=P_{0}=1.013 \times 10^{5} \mathrm{~Pa}$. Note that the pressure does not enter explicitly in Eq. (1), but the equilibrium position $x_{e}$ depends on it (because $\xi_{e}$ depends on the pressure). By increasing the pressure up to a value $P^{*}$, the tube is driven to the situation shown in Fig. $1(\mathrm{c})$, that is, with its top level to the surface $\left(x_{e}=0\right)$. If the pressure is further increased, the tube sinks, as in Fig. 1(d). 


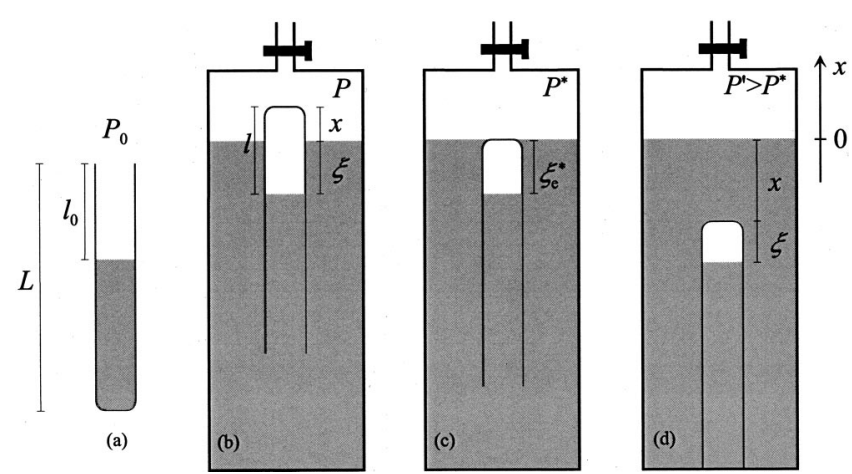

Fig. 1. Schematic of our Cartesian diver experiment. (a) Tube partially filled with water at atmospheric pressure. (b) Tube inverted in a large vessel, where the pressure can be controlled. Our $1 \mathrm{~m}$ high vessel had a cylindrical shape with a diameter of $15 \mathrm{~cm}$. The pressure was regulated with a syringe and measured with a mercury manometer. (c) Tube in static equilibrium with its upper part at the same level as the water in the vessel, a situation that may be achieved by increasing the pressure. (d) By further increasing the pressure the tube sinks.

The position $x_{e}=0$ [see Fig. 1(c)] corresponds to the following air length:

$$
\xi_{e}^{*}=\frac{V}{A}\left(\frac{\rho_{\text {glass }}}{\rho}-1\right)=L\left(\frac{d_{\text {ext }}^{2}}{d_{\text {int }}^{2}}-1\right)\left(\frac{\rho_{\text {glass }}}{\rho}-1\right) .
$$

This quantity depends only on geometrical parameters and on the two densities. It does not even depend on $l_{0}$. If $x_{e}$ $\ll L$ (as was always the case in our experimental conditions), the term in parentheses in Eq. (1) is approximately unity, and we conclude that $\xi_{e} \approx \xi_{e}^{*}$ (as we experimentally observed for our tubes).

If we assume that the air inside the tube behaves as an ideal gas, and that the temperature is the same in cases (a) and (b) of Fig. 1, we have, according to Pascal's principle and Boyle's law,

$$
P_{0} l_{0} A=\left(P+\xi_{e} \rho g\right)\left(\xi_{e}+x_{e}\right) A,
$$

so that

$$
x_{e}=\frac{P_{0} l_{0}}{P+\xi_{e} \rho g}-\xi_{e} .
$$

From Eq. (3) with $x_{e}=0$ (then $\left.P=P^{*}\right)$, we have

$$
P_{0} l_{0}=\left(P^{*}+\xi_{e}^{*} \rho g\right) \xi_{e}^{*} .
$$

In our experiments we always had $\xi_{e} \rho g \ll P$ and, in such a case, the last term in Eq. (5) may be neglected in comparison with the previous one, yielding

$$
\frac{P^{*}}{P_{0}} \approx \frac{l_{0}}{\xi_{e}^{*}}
$$

Table II. Data for type 2 in Table I. Given are the initial air length, $l_{0}$, the pressure increment with respect to the normal atmospheric pressure, $\Delta P$ $=P^{*}-P_{0}$, needed to bring the tube to the position $x_{e}=0\left(\xi_{e}=\xi_{e}^{*}\right)$ [Fig. $1(\mathrm{c})]$, and the ratios $l_{0} / \xi_{e}^{*}$ and $P^{*} / P_{0}$ [the first given by Eq. (6) and the second by direct measurement].

\begin{tabular}{lrcc}
\hline \hline$l_{0}(\mathrm{~cm})$ & $\Delta P(\mathrm{~mm} \mathrm{Hg})$ & $l_{0} / \xi_{e}^{*}[$ Eq. (6) & $P^{*} / P_{0}(\mathrm{expt})$ \\
\hline $4.0 \pm 0.1$ & $-284 \pm 2$ & $0.61 \pm 0.03$ & $0.626 \pm 0.005$ \\
$5.0 \pm 0.1$ & $-210 \pm 2$ & $0.76 \pm 0.03$ & $0.724 \pm 0.005$ \\
$6.0 \pm 0.1$ & $-50 \pm 2$ & $0.92 \pm 0.03$ & $0.934 \pm 0.005$ \\
$7.0 \pm 0.1$ & $90 \pm 2$ & $1.07 \pm 0.03$ & $1.118 \pm 0.005$ \\
$8.0 \pm 0.1$ & $204 \pm 2$ & $1.22 \pm 0.03$ & $1.268 \pm 0.005$ \\
$8.5 \pm 0.1$ & $250 \pm 2$ & $1.30 \pm 0.03$ & $1.328 \pm 0.005$ \\
$9.0 \pm 0.1$ & $284 \pm 2$ & $1.37 \pm 0.03$ & $1.373 \pm 0.005$ \\
\hline \hline
\end{tabular}

In Table I we give the data for different tubes and compare the measured lengths, $\xi_{e}^{*}$, with the values resulting from Eq. (2). The two values agree within the experimental uncertainty. In Table II we compare the ratios $P^{*} / P_{0}$ obtained from the measured air lengths in a particular tube, as given by Eq. (6), and from direct pressure measurements. Again, we find good agreement.

\section{DIVER POTENTIAL AND THE FOLD CATASTROPHE}

When the tube is in static equilibrium, $x=x_{e}$ is given by Eq. (4). If the equilibrium is disturbed, a vertical force appears. From Archimedes' principle, the force is given by

$$
F=\left\{\begin{array}{l}
A \xi \rho g+V(1-x / L) \rho g-V \rho_{\text {glass }} g, \quad x>0 \\
A \xi \rho g+V\left(\rho-\rho_{\text {glass }}\right) g, \quad x \leqslant 0 .
\end{array}\right.
$$

On the other hand, assuming constant temperature, Pascal's principle and Boyle's law yield

$$
P_{0} l_{0}= \begin{cases}(P+\xi \rho g)(\xi+x), & x>0 \\ {[P+(\xi+|x|) \rho g] \xi,} & x \leqslant 0\end{cases}
$$

and the dependence $\xi=\xi(x)$ is obtained from

$$
\xi=\frac{-b^{\prime}+\sqrt{b^{\prime 2}-4 a^{\prime} c^{\prime}}}{2 a^{\prime}}
$$

where

\begin{tabular}{|c|c|c|c|c|c|c|c|}
\hline Tube & $L$ & $m$ & $d_{\text {int }}$ & $d_{\text {ext }}$ & $\rho_{\text {glass }}$ & $\xi_{e}^{*}[$ Eq. (2)] & $\xi_{e}^{*}(\operatorname{expt})$ \\
\hline 1 & $9.8 \pm 0.1$ & $11.9 \pm 0.1$ & 1.380 & 1.600 & $2.35 \pm 0.19$ & $4.57 \pm 0.12$ & $4.6 \pm 0.2$ \\
\hline 2 & $16.0 \pm 0.2$ & $18.6 \pm 0.2$ & 1.430 & 1.640 & $2.29 \pm 0.19$ & $6.51 \pm 0.18$ & $6.4 \pm 0.2$ \\
\hline 3 & $17.1 \pm 0.1$ & $142.8 \pm 0.1$ & 4.440 & 4.970 & $2.16 \pm 0.03$ & $4.96 \pm 0.03$ & $4.9 \pm 0.2$ \\
\hline 4 & $20.1 \pm 0.2$ & $33.3 \pm 0.2$ & 1.740 & 2.000 & $2.17 \pm 0.14$ & $7.55 \pm 0.15$ & $7.6 \pm 0.2$ \\
\hline
\end{tabular}

$$
a^{\prime}=\rho g, \quad b^{\prime}=P+|x| \rho g, \quad c^{\prime}=\left\{\begin{array}{l}
P x-P_{0} l_{0}, \quad x>0 \\
-P_{0} l_{0}, \quad x \leqslant 0
\end{array}\right.
$$

(the other root has no physical meaning). If we substitute Eq. (9) into Eq. (7) and integrate, we obtain the potential:

Table I. Data for various floating tubes. Given are the length, mass, internal and external diameters, the glass mass density, and the air length when the top of the tube is in equilibrium and level with the water surface [see Fig. 1(c)]. CGS units are used. Results from Eq. (2) are compared with direct measurements (expt). 


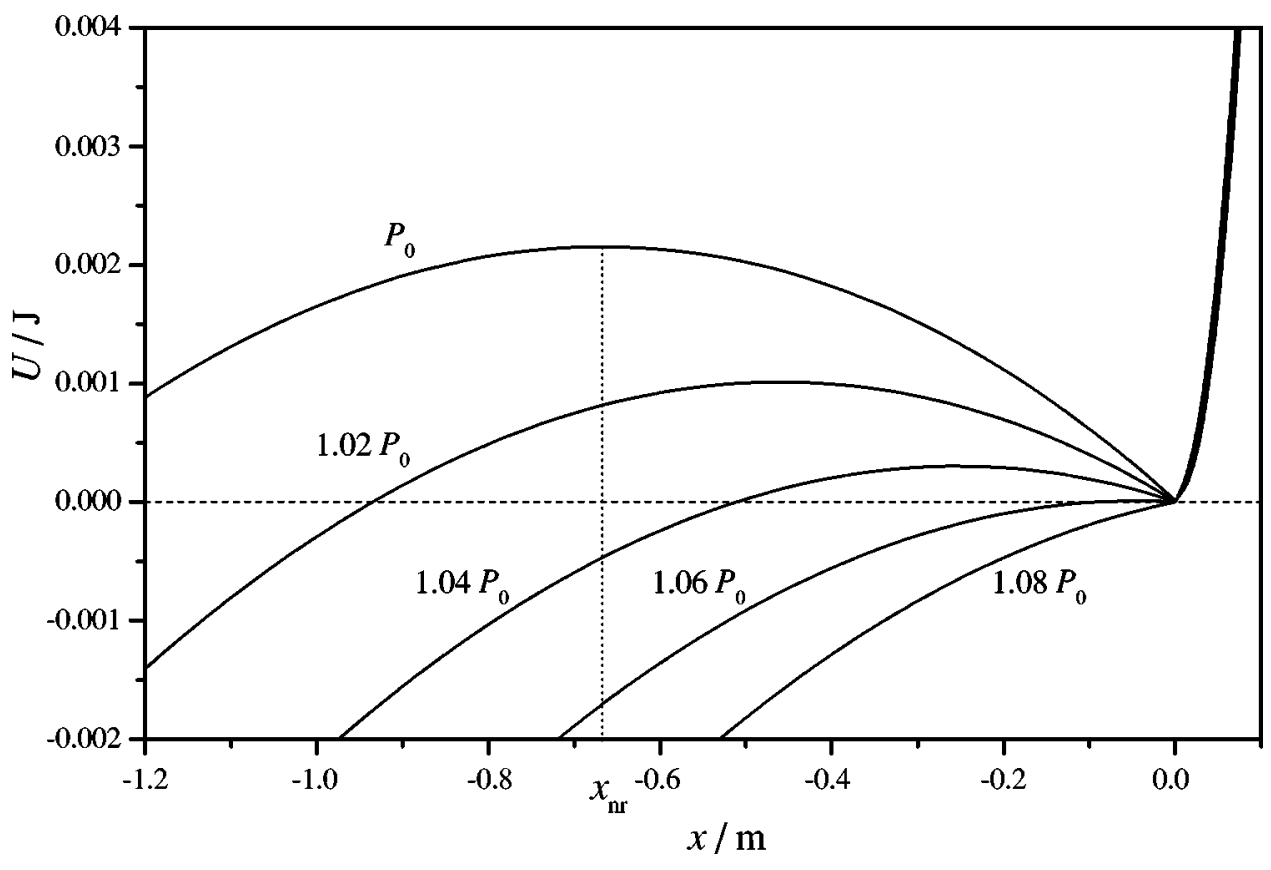

Fig. 2. Cartesian diver potential, Eq. (11), for a tube of type 2 (see Table I) with $l_{0}=7 \mathrm{~cm}$, for various pressures. The no return point, $x_{\mathrm{nr}}$, is indicated for normal atmospheric pressure, $P_{0}$. The critical pressure is $P^{*}=1.07 P_{0}$.

$$
U(x)=\left\{\begin{array}{l}
\frac{\rho g}{2}\left(\frac{A}{2}+\frac{V}{L}\right) x^{2}+\left[\frac{A P}{2}+V\left(\rho_{\text {glass }}-\rho\right) g\right] x-\frac{A}{2} f(x) \\
\quad+C_{1}, \quad x>0 \\
-\frac{A \rho g}{4} x^{2}+\left[\frac{A P}{2}+V\left(\rho_{\text {glass }}-\rho\right) g\right] x-\frac{A}{2} f(x) \\
\quad+C_{2}, \quad x \leqslant 0,
\end{array}\right.
$$

where $C_{1}$ and $C_{2}$ are chosen so that $U\left(x_{e}\right)=0$ and $U(x)$ is continuous across $x=0$, respectively, and $f(x)$ is given by

$$
\begin{aligned}
f(x)= & \int d x \sqrt{(P-\rho g x)^{2}+4 \rho g P_{0} l_{0}} \\
= & -\left(\frac{P}{2 \rho g}-\frac{x}{2}\right) \sqrt{(P-\rho g x)^{2}+4 \rho g P_{0} l_{0}} \\
& +2 P_{0} l_{0} \ln \left[2 \sqrt{(P-\rho g x)^{2}+4 \rho g P_{0} l_{0}}\right. \\
& -2(P-\rho g x)] .
\end{aligned}
$$

The potential is shown in Fig. 2 for various pressures. For sufficiently low pressures the potential has a local minimum at a small positive $x$ value, and a local maximum in the negative region at $x_{\mathrm{nr}}$ (the no return point). For $x>0$ the potential is approximately quadratic. As the pressure increases up to a critical value, the maximum approaches and merges into the minimum at $x=0$, which is an inflection point. Thereafter the potential becomes a monotonic increasing function of $x$. For such a critical pressure, $P^{*}$, a static floating diver is obliged to sink. For $P<P^{*}$, the diver may perform oscillations around the local minimum, but if it crosses the no return point, it is bound to sink.

We may interpret the diver's behavior in the framework of catastrophe theory. The fold catastrophe models the behavior of systems of a single variable and a single control parameter. The generic form of the fold catastrophe potential is $U(x)=x^{3}+a x$, where $a$ is the control parameter. ${ }^{8}$ For these systems there is a discontinuous transition when the control parameter becomes larger than a critical value, $a^{*}=0$. For $a<a^{*}$, the potential has a local maximum and a local minimum. The system has a stable equilibrium point at $x_{e}$ $=\sqrt{|a| / 3}$ (minimum of $U$ ) and an unstable equilibrium point at $x_{e}^{\prime}=-\sqrt{|a| / 3}$ (maximum of $U$ ). In the region $-\infty<x$ $<-\sqrt{|a| / 3}$, there are no equilibrium positions, and $x_{e}^{\prime}$ is a no return point. For suitable initial conditions, the system remains in the region $x>x_{e}^{\prime}$, and if there is a slight friction, it goes eventually to the minimum (with no friction, the system oscillates around the minimum). Keeping the control parameter fixed, the system reaching the region $x<x_{e}^{\prime}$ (with zero or negative velocity) will never return to the region $x>x_{e}^{\prime}$. At the critical value of the control parameter, the maximum and the minimum merge to an inflection point. Beyond the critical value, $a>a^{*}$, there are no equilibrium states: $U(x)$ is an increasing function of $x$.

The Cartesian diver shows a similar behavior, where $\Delta P$ $=P_{0}-P^{*}$ plays the role of the control parameter. If the pressure is kept fixed, but the temperature changes, ${ }^{4}$ a similar behavior may be observed, the temperature now being the control parameter.

The fact that, in practice, all Cartesian divers are inside a vessel with a finite height allows us to explore another point, namely the "constraint catastrophe." 10 Suppose that the vessel is only $0.4 \mathrm{~m}$ high (ours is $1 \mathrm{~m}$ high). The critical pressure for the diver (initially in static equilibrium) to sink is $1.07 P_{0}$ (see Fig. 2). So, for higher pressures, the diver sinks and remains at the bottom of the vessel [as in Fig. 1(d)]. Suppose now that we decrease the pressure. At some value of $P$, the slope of the potential $U(x)$ becomes negative for $x=0.4 \mathrm{~m}$ and the diver comes to the top. For the data of Fig. 2, this pressure is $1.026 P_{0}$. If the vessel is high enough, such a pressure does not exist, and there is no way to bring the diver to the top by external control of the pressure.

Finally, let us mention that, had we maintained the air bubble at constant size, which may be done by closing the tube at stage (a) in Fig. 1, for example, with scotch tape, 


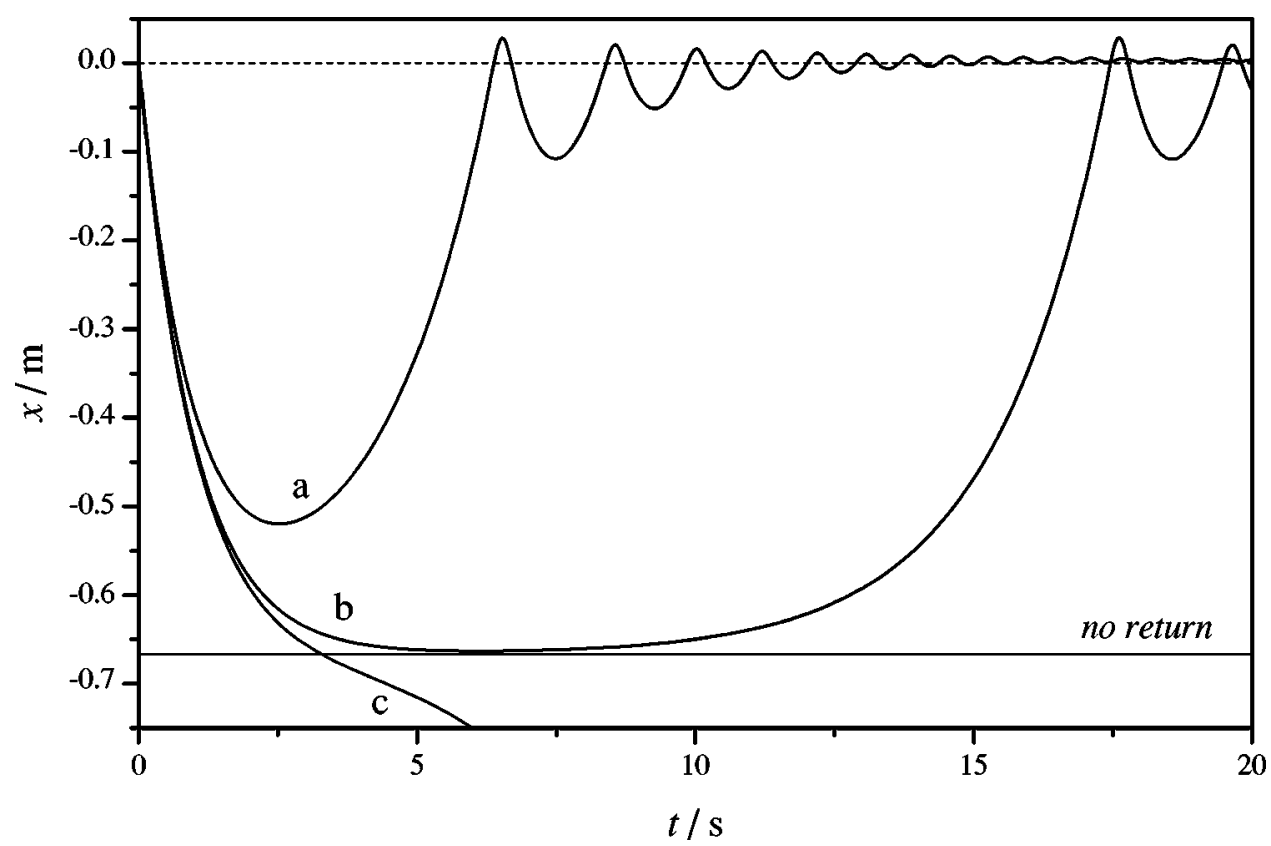

Fig. 3. Position vs time for a tube of type 2 (see Table I) with $l_{0}=7 \mathrm{~cm}$. The pressure is $P=P_{0}=1.013$ $\times 10^{5} \mathrm{~Pa}$. The curves refer to three different initial velocities (a) $v(0)$ $=-0.650 \mathrm{~m} / \mathrm{s}, \quad$ (b) $\quad v(0)=-0.693$ $\mathrm{m} / \mathrm{s}$, (c) $v(0)=-0.700 \mathrm{~m} / \mathrm{s}$, with the friction parameter $b=0.01 \mathrm{~N} \mathrm{~s} \mathrm{~m}^{-1}$. The initial position is the equilibrium position $x_{e}=3,2 \mathrm{~mm}$ [given by Eq. (4)], indicated by the dashed line. The horizontal solid line indicates the no return depth.

before inverting the tube in the vessel, the tube would oscillate around its equilibrium position without any catastrophe. In this case the potential has an absolute minimum.

\section{DIVER DYNAMICS}

We now consider the dynamics of the Cartesian diver for fixed pressure. Because friction is present, Newton's second law is expressed as

$m \frac{d^{2} x}{d t^{2}}=\left\{\begin{array}{l}A \xi(x) \rho g+V(1-x / L) \rho g-V \rho_{\text {glass }} g-b v, \quad x>0 \\ A \xi(x) \rho g+V \rho g-V \rho_{\text {glass }} g-b v, \quad x \leqslant 0,\end{array}\right.$

where a frictional force proportional to the velocity $v$ $=d x / d t$ ( $b$ is the friction parameter) was added to Eq. (7). It is enough to consider a frictional force linear in the velocity because the velocities are always small.

Equation (13) was integrated using an intermediate step algorithm: ${ }^{11-13}$

$$
\begin{aligned}
& v_{n+1 / 2}=v_{n}+a_{n} \frac{\Delta t}{2}, \\
& x_{n+1 / 2}=x_{n}+v_{n+1 / 2} \frac{\Delta t}{2}, \\
& x_{n+1}=x_{n+1 / 2}+v_{n+1 / 2} \frac{\Delta t}{2}, \\
& v_{n+1}=v_{n+1 / 2}+a_{n+1 / 2} \frac{\Delta t}{2} .
\end{aligned}
$$

Figure 3 represents the motion of the Cartesian diver in three cases, with the same initial position, the equilibrium position given by Eq. (4), the same friction parameter $b=0.01 \mathrm{~N} \mathrm{sm}^{-1}$, and the initial velocities (a) $v(0)$ $=-0.650 \mathrm{~m} / \mathrm{s}, \quad$ (b) $v(0)=-0.693 \mathrm{~m} / \mathrm{s}$, and (c) $v(0)$ $=-0.700 \mathrm{~m} / \mathrm{s}$. Figure 3 also shows the no return depth. Once this depth is reached [case (c)], the diver goes all the way down. Similar kinematics is obtained if we keep the same initial conditions (position and velocity), but use different pressures.

In our experiments $x_{e} \simeq 0$, so that the diver was generally submersed, ruled by the second equation of (13). As the diver sinks, the air size decreases, and the force in Eq. (7) may become negative. If

$$
\xi(t)<\frac{V}{A}\left(\frac{\rho_{\text {glass }}}{\rho}-1\right)=\xi_{e}^{*}
$$

[see Eq. (2)], the force acting on the diver points down and the tube keeps sinking. The no return depth, $\left|x_{\mathrm{nr}}\right|$, may be obtained from the second equation of (8), making $x=x_{\mathrm{nr}}$ and $\xi=\xi_{e}^{*}[$ value of $\xi$ when $F=0$, see Eq. (15)]:

$$
\left|x_{\mathrm{nr}}\right|=\frac{1}{\rho g}\left(\frac{P_{0} l_{0}}{\xi_{e}^{*}}-P\right)-\xi_{e}^{*} .
$$

At the initial stage [Fig. 1(b)], we always have $P=P_{0}$. Hence, using Eq. (6), we have

$$
\left|x_{\mathrm{nr}}\right|+\xi_{e}^{*}=\frac{\Delta P}{\rho g}
$$

where $\Delta P=P^{*}-P_{0}$. In Table III we list the measured val-

Table III. The pressure increment, $\Delta P=P^{*}-P_{0}$, and the no return depth (bottom of the air bubble as reference point), $\left|x_{\mathrm{nr}}\right|+\xi_{e}^{*}$, as given by Eq. (17) and experiment for tubes of type 2 (see Table I). The measurements were performed by attaching a small metallic clip to the test tube and using a magnet to move it up and down to detect the unstable equilibrium point. For tubes of type $2, \xi_{e}^{*}=6.51 \pm 0.18 \mathrm{~cm}$ (see Table I).

\begin{tabular}{ccc}
\hline \hline$\Delta P(\mathrm{~mm} \mathrm{Hg})$ & $\left|x_{\mathrm{nr}}\right|+\xi_{e}^{*}[\mathrm{Eq} .(17)]$ & $\left|x_{\mathrm{nr}}\right|+\xi_{e}^{*}(\mathrm{expt})$ \\
\hline $8 \pm 2$ & $11 \pm 3$ & $10.0 \pm 0.5$ \\
$23 \pm 2$ & $31 \pm 3$ & $32.0 \pm 0.5$ \\
$32 \pm 2$ & $43 \pm 3$ & $39.5 \pm 0.5$ \\
$40 \pm 2$ & $54 \pm 3$ & $51.5 \pm 0.5$ \\
$44 \pm 2$ & $60 \pm 3$ & $57.0 \pm 0.5$ \\
$55 \pm 2$ & $75 \pm 3$ & $72.5 \pm 0.5$ \\
$63 \pm 2$ & $86 \pm 3$ & $82.0 \pm 0.5$ \\
\hline
\end{tabular}


ues for $\Delta P$ and the corresponding values for $\left|x_{\mathrm{nr}}\right|+\xi_{e}^{*}$ calculated from Eq. (17). The use of Eq. (16) (with $P=P_{0}$ ), for obtaining $\left|x_{\mathrm{nr}}\right|+\xi_{e}^{*}$ from direct measurements of $l_{0}$, was inconvenient because the range for $l_{0}$ turned out to be too narrow if $\left|x_{\mathrm{nr}}\right|<1 \mathrm{~m}$, as required by our experimental setup. In another set of experiments we measured directly $\left|x_{\mathrm{nr}}\right|$ $+\xi_{e}^{*}$ for the external pressure $P_{0}$ (see the last column in Table III). We found good agreement.

\section{CONCLUSIONS}

We performed a theoretical and experimental study of the Cartesian diver using test tubes with trapped air bubbles. We observed that the floating equilibrium of the diver is no longer an energy minimum when the external pressure is changed, and that the diver placed below a certain depth, which depends on the pressure, never returns to the surface. These surprising results arise simply from a combination of Boyle's law and Pascal's principle, which determine the length of the air column inside the tube and from Archimedes' principle, which determines the buoyancy.

The system is described by a fold catastrophe potential. Thus, a floating diver, at a given critical pressure, sinks spontaneously. If the vessel is high enough, the sunk diver will never come up, even if the pressure is reduced. This irreversible behavior of the fold catastrophe contrasts with the reversible character of cusp catastrophes (usually with hysteresis). As far as we know, this is one of the few physical examples showing a fold catastrophe behavior (a situation described in Ref. 14 is another example).

\footnotetext{
a)Electronic mail: guemezj@unican.es

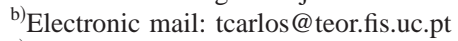

${ }^{c)}$ Electronic mail: tmanuel@teor.fis.uc.pt

${ }^{1}$ R. C. Turner, "Toys in teaching: Cartesian diver," Am. J. Phys. 51, 475-

476 (1983); R. N. Jones, "The Cartesian diver," Phys. Teach. 11, 345
}

(1973). Some comments about this toy can be found in W. A. Butler, "Reverse Cartesian diver 'trick,', Am. J. Phys. 49, 92 (1981); M. Gardner, "The Cartesian matches," Phys. Teach. 28, 478 (1990); P. Kirkpatrick, "A neglected lesson from the Cartesian diver," Am. J. Phys. 10, 160 (1942); R. F. Kotheimer, "Cartesian diver," Phys. Teach. 12, 576 (1974); H. Kruglak, "The rising Cartesian diver," ibid. 13, 68-69 (1975); R. S. Mackey, "Automatic Cartesian diver," Am. J. Phys. 26, 403-404 (1958); J. S. Miller, "Extension of the Cartesian diver experiment," ibid. 22, 235236 (1954); L. P. Orwig, "Cartesian diver 'tricks'," ibid. 48, 320 (1980); R. L. Wild, "Ultimate Cartesian diver set," ibid. 49, 1185 (1981).

${ }^{2}$ R. M. Graham, “An extremely sensitive Cartesian diver,” Phys. Teach. 32, 182-183 (1994).

${ }^{3}$ R. M. Cosby and D. E. Petry, "Simple buoyancy demonstrations using saltwater," Phys. Teach. 27, 550-551 (1989).

${ }^{4}$ K. D. Pinkerton, "Sink or swim: The Cartesian diver," J. Chem. Educ. 78, 200A (2001).

${ }^{5}$ E. V. Lee, "Cartesian diver with pressure head," Phys. Teach. 19, 416 (1981).

${ }^{6}$ S. He, S. Mak, and E. Zhu, "Depth dependent Cartesian divers," Am. J. Phys. 61, 938-940 (1993).

${ }^{7} \mathrm{R}$. Gilmore, Catastrophe Theory for Scientist and Engineers (Wiley, New York, 1981)

${ }^{8}$ P. T. Saunders, An Introduction to Catastrophe Theory (Cambridge U.P., New York, 1980).

${ }^{9}$ Neglecting the volume at the end of the tube, which is approximately $3 \%$ of the total volume.

${ }^{10}$ T. Poston and I. Stewart, Catastrophe Theory and its Applications (Pitman, London, 1978).

${ }^{11}$ This algorithm is a modification of the velocity form of the Verlet algorithm given for example in Eq. (5.45) of Ref. 12 with $a_{n+1 / 2}$ in the last expression of Eq. (14) replaced by $a_{n+1}$. This modified form of the Verlet algorithm is required if the force depends on the velocity. See Ref. 13 for a discussion of the algorithm.

${ }^{12} \mathrm{H}$. Gould and J. Tobochnik, An Introduction to Computer Simulation Methods (Addison-Wesley, Reading, MA, 1996), 2nd ed.

${ }^{13}$ J. Dias de Deus, M. Pimenta, A. Noronha, T. Peña, and P. Brogueira, Introdução à Física (McGraw-Hill, Portugal, Lisbon, 2000), 2nd ed.

${ }^{14}$ P. Gnädig, G. Honyek, and K. Riley, 200 Puzzling Physics Problems with Hints and Solutions (Cambridge U.P., Cambridge, 2001). In Problem P27, pp. 6,52 , and $86-88$, a system is described that also presents a fold catastrophe.

\section{LOOKING DOES NOT ALWAYS LEAD TO LEARNING}

Children can look at pendulums, as children and adults did for thousands of years, without seeing any of the scientific properties of the pendulum, without experiencing any dissonance. They will begin to have knowledge of the pendulum not when they look and discuss, but when they are effectively taught by teachers who have mastered the science and mathematics of pendulum motion and can convey this knowledge in a meaningful and engaging manner. This could mean giving lectures, setting pages of text to read, organizing practical work and setting investigative questions, arranging small group discussions and so on.

Michael R. Matthews, Time for Science Education: How Teaching the History and Philosophy of Pendulum Motion Can Contribute to Science Literacy (Kluwer Academic/Plenum Publishers, New York, NY, 2000), p. 281.

Submitted by Alan J. DeWeerd. 